Received: June 16, 2010

Accepted after revision: August 6, 2010

Published online: February 2, 2011

\title{
Subcutaneous Immunotherapy with a Depigmented Polymerized Birch Pollen Extract - A New Therapeutic Option for Patients with Atopic Dermatitis
}

\author{
Natalija Novak ${ }^{a}$ Diamant Thaci ${ }^{b}$ Matthias Hoffmann ${ }^{c}$ Regina Fölster-Holst ${ }^{d}$ \\ Thilo Biedermann $^{\text {e }}$ Bernhard Homey ${ }^{f}$ Knut Schaekelg Josef A. Stefan ${ }^{\text {h }}$ \\ Thomas Werfel $^{i}$ Thomas Bieber ${ }^{a} \quad$ Angelika Sager ${ }^{j}$ Torsten Zuberbierk \\ ${ }^{a}$ Department of Dermatology and Allergy, University of Bonn, Bonn, ${ }^{b}$ Department of Dermatology, University of \\ Frankfurt, Frankfurt, ' Dermatology Practice, Witten, ${ }^{\mathrm{d}}$ Department of Dermatology and Venerology, University \\ Hospital Schleswig-Holstein, Campus Kiel, University of Kiel, Kiel, e Department of Dermatology, University \\ of Tübingen, Tübingen, ${ }^{f}$ Department of Dermatology, University of Düsseldorf, Düsseldorf, ${ }^{9}$ Department of \\ Dermatology, University of Dresden, Dresden, h Dermatology Practice, Hennef, i'Department of Dermatology, \\ Medical University of Hannover (MHH), Hannover, ${ }^{\mathrm{j}}$ Leti Pharma, Witten, and ${ }^{\mathrm{k}}$ Department of Dermatology and \\ Allergy Charité, Allergy Centre Charité, Universitätsmedizin Berlin, Berlin, Germany
}

\section{Key Words}

Atopic dermatitis · Birch pollen · Specific immunotherapy

\begin{abstract}
Background: Birch pollen is an important outdoor allergen able to aggravate symptoms in atopic dermatitis (AD). Specific immunotherapy (SIT), an established procedure for allergic airway diseases, might also represent an attractive therapeutic option for the causal treatment of allergen-triggered cutaneous symptoms in these patients. Studies with house dust mite SIT have already shown beneficial effects in AD patients, whereas the safety and efficacy of SIT with birch pollen extract in AD patients have not been studied so far. The aim of this study was to evaluate for the first time the safety and efficacy of SIT with a depigmented polymerized birch pollen extract in AD patients. Methods: Fifty-five adult
\end{abstract}

patients with moderate-to-severe $A D$ and clinically relevant sensitization to birch pollen received SIT for 12 weeks. SIT was continued during birch pollen season. The assessment of safety, the total SCORAD value, and the Dermatology Life Quality Index (DLQI) were evaluated. Results: The median total SCORAD value was reduced by $34 \%(p<0.001)$ during the course of treatment and the mean DLQI improved by $49 \%(p<0.001)$ despite strong simultaneous birch pollen exposure. Eight patients (14.5\%) developed systemic reactions and 19 patients (34.5\%) developed local reactions which were of mild intensity in most cases. No patient discontinued the study prematurely due to adverse drug reactions. Coseasonal treatment was well tolerated. Conclusion: SIT with a depigmented polymerized birch pollen extract leads to significant improvement of the SCORAD value and the DLQI in patients suffering from moderate-to-severe $A D$ sensitized to birch pollen.
Copyright $\odot 2011$ S. Karger AG, Basel

\section{KARGER}

Fax +41613061234 E-Mail karger@karger.ch www.karger.com
(C) 2011 S. Karger AG, Basel

$1018-2438 / 11 / 1553-0252 \$ 38.00 / 0$

Accessible online at:

www.karger.com/iaa
Correspondence to: Dr. Natalija Novak

Department of Dermatology and Allergy, University of Bonn

Sigmund-Freud-Strasse 25, DE-53105 Bonn (Germany)

Tel. +49 228287 15370, Fax +49228 28714333

E-Mail Natalija.Novak@ukb.uni-bonn.de 


\section{Introduction}

To date, therapy for atopic dermatitis (AD), a frequent chronic inflammatory skin disease, is limited to symptomatic anti-inflammatory, antipruritic, or immunomodulatory treatment approaches [1]. However, rationale-based treatment conducted to counteract diseaseaggravating pathways induced by trigger factors for $\mathrm{AD}$ would be much more effective in reducing the number of flare-ups and the severity of $\mathrm{AD}$, thereby improving the quality of life of these patients. Although it is still unclear whether allergic sensitizations mirrored by elevated total serum immunoglobulin E (IgE) and allergen-specific IgE levels detectable in a majority of $\mathrm{AD}$ patients represent primary or secondary disease-related factors, indoor allergens such as house dust mites (HDM) as well as seasonal allergens including birch and grass pollen allergens have been clearly shown to promote exacerbations and impairment of the disease [2].

Allergens represent important triggers in a majority of AD patients. Allergen-specific immunotherapy is successfully in use as a long-term treatment of sensitizations in patients with related atopic disorders such as rhinitis and mild asthma. The efficacy of specific immunotherapy (SIT) in rhinitis and mild asthma is well proven and is related to immunologic changes such as a shift of Th2 immune responses into modified Th1 immune responses as well as the induction of regulatory $\mathrm{T}$ cells and other tolerogenic pathways $[3,4]$. Thus, the first open-label and controlled studies on SIT have been conducted in AD patients with sensitizations to HDM. Altogether, most of these studies have provided very promising results $[5,6]$. However, most of the controlled and uncontrolled studies published on this topic so far have focused on the treatment of sensitizations to HDM or grass pollen allergens in $\mathrm{AD}$ patients [7], but none of the studies have investigated the safety and efficacy of SIT in AD patients sensitized to birch pollen allergen. As a consequence, at present there are no data available which would allow any reliable assessment of the value of SIT with birch pollen allergens as a rationale-based treatment approach for $\mathrm{AD}$.

Therefore, we performed a multicenter, open, pilot study in adult patients with AD and clinically relevant sensitizations to birch pollen allergen.

\section{Methods}

Patients and Diagnostic Criteria

A total of $55 \mathrm{AD}$ patients between 18 and 65 years of age with moderate-to-severe $\mathrm{AD}$ diagnosed according to the criteria of
Table 1. Summary of the demographic baseline characteristics

\begin{tabular}{lc}
\hline Demographic variables & Overall $(\mathrm{n}=55)$ \\
\hline Gender & \\
$\quad$ Male, $\mathrm{n}(\%)$ & $20(36.4)$ \\
$\quad$ Female, $\mathrm{n}(\%)$ & $35(63.6)$ \\
Age, years & $36.3 \pm 10.7$ \\
Total serum IgE, $\mathrm{kU} / \mathrm{l}$ & $2,181.6 \pm 4,169.9$ \\
Birch pollen-specific IgE, $\mathrm{kU} / \mathrm{l}$ & $77.8 \pm 32.9$ \\
\hline
\end{tabular}

Values are presented as means \pm SD unless otherwise specified.

Hanifin and Rajka were included. Disease severity was assessed using a severity scoring of atopic dermatitis (SCORAD) [8]. Patients with a SCORAD value $\geq 25$ and a duration of eczema longer than 2 years were selected. Birch pollen sensitization was assessed by specific serum IgE against birch pollen in CAP-RAST $\geq 3$, a positive atopy patch test, and/or a positive skin prick test for birch pollen allergen as well as symptoms of AD related to birch pollen exposure. The demographic data of the study cohort are summarized in table 1 . Of the $\mathrm{AD}$ patients, $10.9 \%$ had concomitant allergic asthma and $5.5 \%$ had allergic rhinoconjunctivitis.

Obligatory exclusion criteria were: $\mathrm{FEV}_{1}<70 \%$ of the predicted value measured by the peak flow; SIT against Betula Verrucosa (Bet $v 1$; birch pollen) during the last 5 years; pretreatment with systemic corticosteroids, immunosuppressive agents or UV therapy 1 month before SIT or during SIT; acute tuberculosis; inflammatory or infectious diseases of the target organ; immunopathological diseases in which autoimmune mechanisms play a role; immune deficiencies; treatment with $\beta$-antagonists; any disease prohibiting the use of adrenaline; serious psychiatric and psychological disturbances; concomitant treatment with substances interfering with the immune system; pregnancy; immunization with vaccines 7 days prior to SIT and 14 days post-SIT, and acute and chronic eczema at the skin prick test site.

Study Design

The study was designed as an open-label pilot study to assess the safety and efficacy of SIT with depigmented birch pollen extract in AD patients. The study was conducted from January 2008 to January 2009. The regional pollen count measurement was documented at each center. The use of concomitant medications with emollients and topical and systemic drugs was regularly documented in each patient. The study was approved by the ethics committee and informed consent was obtained from every individual participating in the study.

Allergen Preparation and Treatment Schedule

The treatment was subdivided into a build-up phase of 3 weeks followed by a maintenance treatment period of 12 weeks with SIT using a depigmented polymerized birch pollen extract. The initial build-up period during the first 3 weeks after screening consisted of weekly administrations of gradually increasing amounts of vial 1 at a concentration of $100 \mathrm{DPP} / \mathrm{mI}$ and vial 2 at a concentration of $1,000 \mathrm{DPP} / \mathrm{ml}$ (1 DPP was the result of depigmenting and po- 
Table 2. Summary of the SIT schedule

\begin{tabular}{llll}
\hline Vial & Injection No. & Interval & Milliliters \\
\hline 1 & 1 & 1 week & 0.2 \\
& 2 & 1 week & 0.5 \\
\hline 2 & 3 & 1 week & 0.2 \\
& 4 & 1 week & 0.5 \\
& $5+6$ & 6 weeks & 0.5 \\
\hline
\end{tabular}

Table 3. Summary of side effects observed during the study

\begin{tabular}{lll}
\hline Symptoms & Patients, $\mathrm{n}(\%)$ & Symptoms, $\mathrm{n}$ \\
\hline Eczematous flare & $2(3.6)$ & 2 \\
Pruritus & $2(3.6)$ & 2 \\
Procedural headache & $1(1.8)$ & 1 \\
Allergic rhinitis & $1(1.8)$ & 1 \\
Urticaria & $1(1.8)$ & 1 \\
Vertigo & $1(1.8)$ & 1 \\
Local reactions & $19(34.5)$ & 36 \\
\hline
\end{tabular}

lymerizing 1 HEPL of birch pollen allergenic extract). Treatment started with 7-day administration intervals of gradually increasing single doses of extract until the recommended dose was reached (i.e. $0.2 \mathrm{ml}$ vial $1,0.5 \mathrm{ml}$ vial $1,0.2 \mathrm{ml}$ vial 2 , and $0.5 \mathrm{ml}$ vial 2). This dose $(0.5 \mathrm{ml}$ of vial 2$)$ was further maintained over the remaining treatment period in 6-week intervals for a total maintenance phase of 12 weeks (table 2). Regular patient visits were before SIT as well as at weeks 1, 2, 3, 9, and 15. A follow-up visit was conducted 2 weeks after the end of SIT. Treatment was started before and continued during the birch pollen season.

\section{Statistical Analysis}

Statistical analysis was performed with SPSS 17.0 for Windows using a t test for normally distributed samples. The calculated values shown are means \pm standard error of the mean (SEM).

\section{Results}

Significant Improvement of the SCORAD Value and the Dermatology Life Quality Index under SIT

The median total SCORAD value decreased by $34 \%$ $(p<0.001)$ during the course of treatment (fig. 1a). The mean Dermatology Life Quality Index (DLQI) improved by $49 \%$ ( $\mathrm{p}<0.001)$, indicating a profound positive influence of SIT not only on the clinical symptoms of $\mathrm{AD}$ but
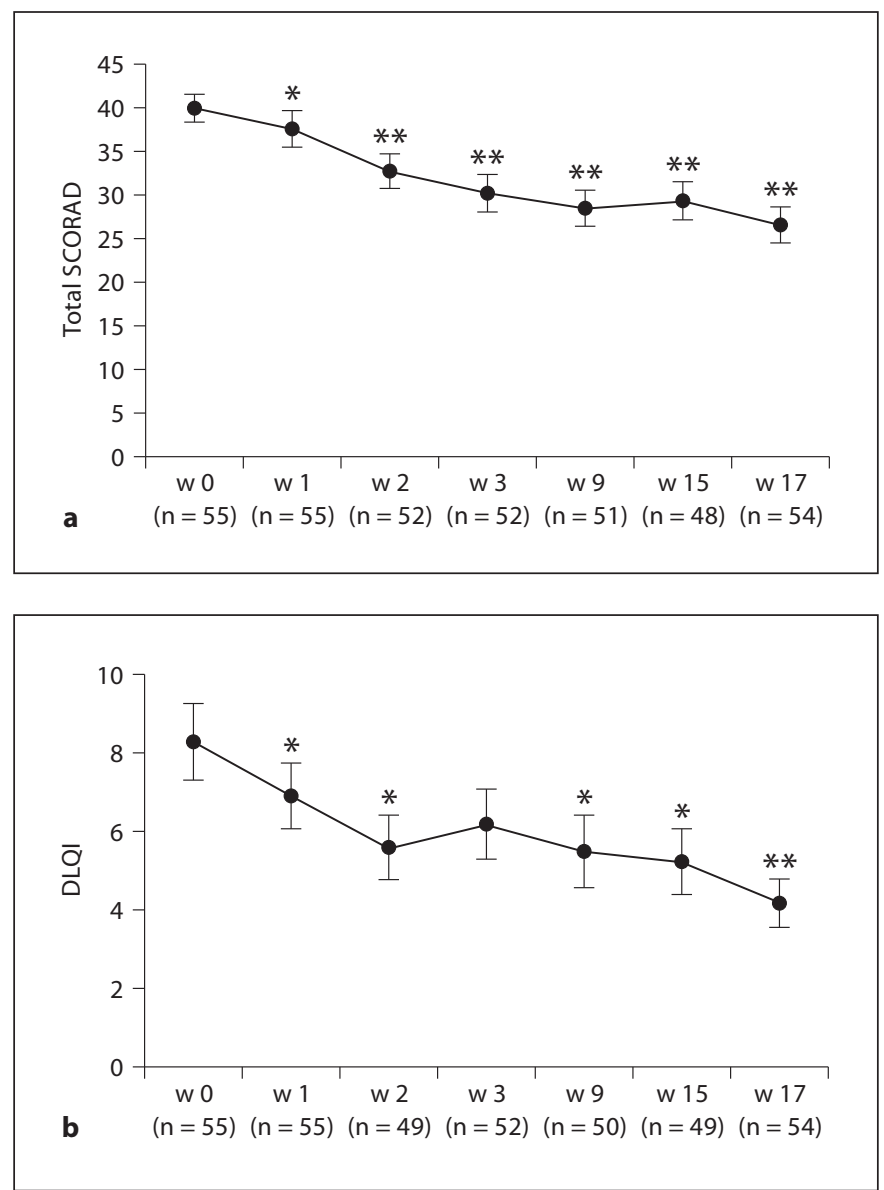

Fig. 1. The SCORAD value and the DLQI decrease significantly under SIT. a The mean values of the total SCORAD value \pm SEM during different weeks of SIT are depicted. $\mathbf{b}$ The mean values of the DLQI \pm SEM during treatment are shown. ${ }^{*} \mathrm{p}<0.05 ;{ }^{* *} \mathrm{p}<$ 0.001 ; no indication $=$ Not significant; $\mathrm{w}=$ week.

also on patients' quality of life (fig. 1b). Furthermore, it is important to note that improvement was already noticeable after the build-up phase, with continuous improvement of the total SCORAD value and the DLQI until the end of treatment.

The Frequency of Side Reactions Was Comparable to Other Studies on SIT with Depigmented Birch Pollen

\section{Extract}

A total of 24 patients developed adverse drug reactions. Eight patients (14.5\%) developed systemic reactions which were mostly of mild intensity and consisted of flare-ups of eczematous (2 patients) or urticarial skin lesions, a worsening of the symptoms of rhinitis, an increase in pruritus, transient headache, or vertigo. Nine- 
teen patients (34.5\%) developed local reactions, $30 \%$ of which occurred immediately after injection. Local side reactions were mostly of mild intensity (table 3 ). No patient discontinued the study prematurely due to adverse drug reactions. Based on a calculated weekly dose, usage of the concomitant medications was constant before, during, and after SIT, indicating that there was no negative influence of SIT on AD. Moreover, the use of systemic antihistamines and nasal sprays decreased during SIT as compared to baseline.

Furthermore, it is important to note that SIT was continued during birch pollen season; thus, the SCORAD value and the DLQI improved despite simultaneous birch pollen exposure. On average, every patient was treated for 19.2 days during birch pollen season. About $60 \%$ of patients reached the maintenance dose until the onset of birch pollen season.

\section{Discussion}

Here, we demonstrated that the treatment of AD patients sensitized to birch pollen with SIT leads to profound improvement of the SCORAD value and the DLQI already within the first few weeks of therapy.

Therefore, this study provides the first evidence of the safety and efficacy of SIT with a depigmented polymerized birch pollen extract in patients with moderate-tosevere $\mathrm{AD}$. These data confirm the conceptual approach of using SIT for the long-term treatment of sensitizations in $\mathrm{AD}$ patients, as has been done in rhinitis and mild asthma for several years now $[9,10]$. Furthermore, the data amend the observations on the safety and efficacy of SIT gained in the context of the treatment of AD patients with sensitizations against HDM. Despite improvement of the skin lesions, a reduction in CCL17, CCL22, and other serum factors known to go along with the severity of $\mathrm{AD}$ has been observed in $\mathrm{AD}$ patients during SIT, with no significant increase in the total and allergen-specific IgE serum levels [11, 12].

Therefore, the results of this study might represent the first steps towards an expansion of the therapeutic options of SIT in AD patients to patients with sensitizations to birch pollen allergen.

When comparing the results of this trial with other open-label or controlled studies on SIT in AD, the relatively short treatment phase of only 12 weeks in this study has to be considered $[5,13]$. This allows a relatively clear assessment of safety but only a preliminary conclusion about the efficacy of this treatment, which is already very satisfactory after 12 weeks but is likely to be even stronger after a longer treatment period.

Since this study was conducted to assess the first data on the safety and efficacy of SIT using a depigmented birch pollen extract in adult patients with $\mathrm{AD}$, an openlabel study design was been selected. Therefore, based on previous studies with comparable invasive treatments, a placebo effect of up to $30 \%$ improvement of AD severity has to be considered. However, the improvement achieved in this study clearly exceeds this effect.

The frequency of local and systemic side reactions in AD patients was comparable to the rate observed in so-far unpublished double-blind placebo-controlled studies on SIT with depigmented birch/tree pollen extract performed in patients with allergic rhinitis.

Despite HDM, birch and grass pollen allergens represent strong exogenous trigger factors in a subgroup of $\mathrm{AD}$ patients. The relevance of birch pollen allergens for eczema development is further documented by a relatively high rate of positive atopy patch test reactions to birch pollen allergens as compared to other aeroallergens such as grass pollen or cat dander in sensitized AD patients [14]. Moreover, the skin of AD patients with high sensitization levels to birch pollen allergens is much more colonized with enterotoxin-producing Staphylococcus aureus bacteria, and those patients were demonstrated to suffer from more severe forms of $\mathrm{AD}$ [15]. In addition, birch pollen-related foods have been observed to provoke flareups of AD as well as the accumulation of birch pollenspecific $\mathrm{T}$ cells in the AD skin lesions of birch pollensensitized patients $[16,17]$. In the long term, treating birch pollen-sensitized AD patients with SIT would not only allow the efficient therapeutic reduction of birch pollen-triggered flare-ups of $\mathrm{AD}$, but it would also putatively impact on the impairment of AD by other cofactors associated with birch pollen sensitizations modifying the severity of AD, such as bacterial colonization an birchrelated food allergens. However, based on the first data on the safety and efficacy of birch pollen SIT in AD presented here, double-blind, placebo-controlled, pivotal studies are required to confirm and further verify the value of birch pollen SIT as an alternative therapeutic option for patients with $\mathrm{AD}$.

\section{Acknowledgements}

The study was funded by LETI Pharma, Witten, Germany. N.N. is supported by a Heisenberg-Professorship of the German Research Council (DFG; NO454/5-2). 


\section{References}

1 Jung T, Stingl G: Atopic dermatitis: therapeutic concepts evolving from new pathophysiologic insights. J Allergy Clin Immunol 2008;122:1074-1081.

$\checkmark 2$ Bieber T, Novak N: Pathogenesis of atopic dermatitis: new developments. Curr Allergy Asthma Rep 2009;9:291-294.

3 Larche M, Akdis CA, Valenta R: Immunological mechanisms of allergen-specific immunotherapy. Nat Rev Immunol 2006;6: 761-771.

4 Calderon MA, Alves B, Jacobson M, Hurwitz B, Sheikh A, Durham S: Allergen injection immunotherapy for seasonal allergic rhinitis. Cochrane Database Syst Rev 2007;1: CD001936.

5 Werfel T, Breuer K, Rueff F, Przybilla B, Worm M, Grewe M, et al: Usefulness of specific immunotherapy in patients with atopic dermatitis and allergic sensitization to house dust mites: a multi-centre, randomized, dose-response study. Allergy 2006;61:202205.

6 Silny W, Czarnecka-Operacz M: Specific immunotherapy in the treatment of patients with atopic dermatitis-results of double blind placebo controlled study (in Polish). Pol Merkur Lekarski 2006;21:558-565.
Bussmann C, Bockenhoff A, Henke H, Werfel T, Novak N: Does allergen-specific immunotherapy represent a therapeutic option for patients with atopic dermatitis? J Allergy Clin Immunol 2006;118:1292-1298.

8 Severity scoring of atopic dermatitis: the SCORAD index. Consensus Report of the European Task Force on Atopic Dermatitis. Dermatology 1993;186:23-31.

-9 Larche M, Akdis CA, Valenta R: Immunological mechanisms of allergen-specific immunotherapy. Nat Rev Immunol 2006;6: 761-771.

10 Bussmann C, Maintz L, Hart J, Allam JP, Vrtala S, Chen KW, et al: Clinical improvement and immunological changes in atopic dermatitis patients undergoing subcutaneous immunotherapy with a house dust mite allergoid: a pilot study. Clin Exp Allergy 2007;37:1277-1285.

11 Frew AJ: Allergen immunotherapy. J Allergy Clin Immunol 2010;125(suppl 2):S306-S313.

12 Kwon YS, Oh SH, Wu WH, Bae BG, Lee HJ, Lee MG, et al: CC chemokines as potential immunologic markers correlated with clinical improvement of atopic dermatitis patients by immunotherapy. Exp Dermatol 2009;19:246-251.
13 Czarnecka-Operacz M, Silny W: Specific immunotherapy in atopic dermatitis - Fouryear treatment in different age and airborne allergy type subgroups. Acta Dermatovenerol Croat 2006;14:230-240.

14 Samochocki Z, Owczarek W, Rujna P, Raczka A: Hypersensitivity to aeroallergens in adult patients with atopic dermatitis develops due to the different immunological mechanisms. Eur J Dermatol 2007;17:520524.

15 Wichmann K, Uter W, Weiss J, Breuer K, Heratizadeh A, Mai U, et al: Isolation of alpha-toxin-producing Staphylococcus aureus from the skin of highly sensitized adult patients with severe atopic dermatitis. Br J Dermatol 2009; 161:300-305.

16 Breuer K, Wulf A, Constien A, Tetau D, Kapp A, Werfel T: Birch pollen-related food as a provocation factor of allergic symptoms in children with atopic eczema/dermatitis syndrome. Allergy 2004;59:988-994.

17 Reekers R, Busche M, Wittmann M, Kapp A, Werfel T: Birch pollen-related foods trigger atopic dermatitis in patients with specific cutaneous T-cell responses to birch pollen antigens. J Allergy Clin Immunol 1999;104: 466-472. 